\title{
1 Antimicrobial prodrug activation by the staphylococcal glyoxalase GloB
}

3 Marwa O. Mikati ${ }^{1^{\star}}$, Justin J. Miller ${ }^{{ }^{\star}}$, Damon M. Osbourn ${ }^{2}$, Naomi Ghebremichael ${ }^{1}$, Ishaan T.

4 Shah $^{1}$, Carey-Ann D. Burnham ${ }^{1,3,4}$, Kenneth M. Heidel ${ }^{5}$, Victoria C. Yan ${ }^{6}$, Florian L. Muller ${ }^{6}$,

5 Cynthia S. Dowd ${ }^{5}$, Rachel L. Edwards ${ }^{1}$, Audrey R. Odom John ${ }^{1,3,7 a}$.

6

7 'Department of Pediatrics, Washington University School of Medicine, St. Louis, MO, USA;

$8 \quad$ 2Department of Molecular Microbiology and Immunology, Saint Louis University School of

9 Medicine, St. Louis, MO, USA; ${ }^{3}$ Department of Molecular Microbiology, Washington University

10 School of Medicine, St. Louis, MO, USA; ${ }^{4}$ Department of Pathology and Immunology,

11 Washington University School of Medicine, St. Louis, MO, USA; ${ }^{5}$ Department of Chemistry, The

12 George Washington University, Washington, DC, USA; ${ }^{6}$ Department of Cancer Systems

13 Imaging, The University of Texas MD Anderson Cancer Center, Houston, TX USA; .

$14{ }^{7}$ Department of Pediatrics, Children's Hospital of Philadelphia, Philadelphia, PA, USA (current

15 institution)

16

17 * M.M. and J.M. contributed equally to this work.

18 a Correspondence and requests for materials should be addressed A.R.O.J.

19 (JOHNA3@email.chop.edu) 


\section{ABSTRACT}

2 With the rising prevalence of multidrug-resistance, there is an urgent need to develop novel

3 antibiotics. Many putative antibiotics demonstrate promising in vitro potency but fail in vivo due

4 to poor drug-like qualities (e.g. serum half-life, oral absorption, solubility, toxicity). These drug-

5 like properties can be modified through the addition of chemical protecting groups, creating

6 "prodrugs" that are activated prior to target inhibition. Lipophilic prodrugging techniques,

7 including the attachment of a pivaloyloxymethyl group, have garnered attention for their ability to

8 increase cellular permeability by masking charged residues and the relative ease of the

9 chemical prodrugging process. Unfortunately, pivaloyloxymethyl prodrugs are rapidly activated

10 by human sera, rendering any membrane permeability qualities absent during clinical treatment.

11 Identification of the bacterial prodrug activation pathway(s) will allow for the development of

12 host-stable and microbe-targeted prodrug therapies. Here, we use two zoonotic staphylococcal

13 species, S. schleiferi and S. pseudintermedius, to establish the mechanism of carboxy ester

14 prodrug activation. Using a forward genetic screen, we identify a conserved locus in both

15 species encoding the enzyme hydroxyacylglutathione hydrolase (GloB), whose loss-of-function

16 confers resistance to carboxy ester prodrugs. We enzymatically characterize GloB and

17 demonstrate that it is a functional glyoxalase II enzyme, which has the capacity to activate

18 carboxy ester prodrugs. As GloB homologs are both widespread and diverse in sequence, our

19 findings suggest that GloB may be a useful mechanism for developing species- or genus-level

20 prodrug targeting strategies. 


\section{INTRODUCTION}

2 In 2019, the United States recorded 2.8 million antibiotic-resistant infections, resulting in over

335,000 deaths (1). The recent surge in antibiotic use in the setting of the COVID-19 pandemic

4 portends an acceleration of the antibiotic resistance threat $(1,2)$. Staphylococcus aureus is a

5 formidable human pathogen that causes a wide variety of invasive and life-threatening

6 infections. Closely related staphylococcal species, S. pseudintermedius and S. schleiferi, cause

7 similar skin, soft tissue, and invasive infections in companion animals and are increasingly

8 appreciated as serious pathogens of humans (3-6). Rising rates of methicillin resistance are

9 reported in all three species, with methicillin-resistant $S$. aureus (MRSA) labeled a "serious

10 threat" by the Centers for Disease Control and Prevention (CDC) (7-10). Novel antimicrobial

11 strategies that circumvent existing drug resistance mechanisms are urgently needed.

13 Bacterial metabolism is a promising area for antimicrobial development $(11,12)$. Many

14 metabolic processes are essential for bacterial growth and pathogenesis. However, targeting

15 metabolic processes can be inherently challenging, as a substantial portion of metabolism

16 involves the catalytic transformation of highly charged substrates (e.g. phosphate transfer

17 reactions). Substrate-competitive inhibitors of metabolic enzymes frequently deploy

18 phosphonate functional groups as isosteric phosphate mimics (13). These negatively charged

19 phosphonate antimetabolite inhibitors are prone to unacceptable drug-like characteristics and

20 often diffuse poorly across membranes (14-19).

22 Prodrugging, or the modification of an inhibitor through addition of labile chemical adducts, is a

23 common medicinal chemistry strategy to improve drug-like properties of an inhibitor under

24 development (19-21). As promoieties are released prior to inhibitor-target engagement,

25 prodrugging can temporarily cloak problematic pharmacokinetic properties such as poor 
1 absorption or solubility. For example, the third-generation cephalosporin, cefditoren, is poorly

2 absorbed in the small intestine unless the phosphonate residues are masked with a lipophilic

3 pivaloyloxymethyl (POM) promoiety, in the form of cefditoren pivoxil (22). Similarly, nucleoside

4 analogues are generally cell-impermeable, but their cognate prodrugs have much improved

5 cellular penetration and antiviral efficacy, as seen in remdesivir (SARS-CoV2), tenofovir

6 disoproxil (HIV), and sofosbuvir (hepatitis C virus, HCV) (23-26). We have recently employed

7 lipophilic prodrugging strategies to increase the efficacy of broad-spectrum antimicrobial

8 phosphonate antibiotics. Notably, POM ester modification of a phosphonate isoprenoid

9 biosynthesis inhibitor (ERJ) increases antistaphyloccal activity by 200- and 500-fold for $S$.

10 schleiferi and S. pseudintermedius, respectively (27). Similar dramatic potency gains are

11 observed for the same class of compounds against Mycobacterium tuberculosis, Yersinia

12 pestis, Franciscella novicida, and the malaria parasite, Plasmodium falciparum (16, 28-31).

14 While pivaloyloxymethyl ester (POM)-prodrugs demonstrate remarkable potency in vitro, POM-

15 promoieties are known to be rapidly hydrolyzed by serum carboxylesterases (32). If cell-

16 impermeable phosphonate antibiotics are to be effective at the site of infection, the promoiety

17 must be resistant to premature bioactivation during absorption and distribution in the circulation.

18 This specificity in prodrug activation has been successfully achieved for liver-targeted prodrugs,

19 using the "HepDirect" prodrug approach, but has not yet been deployed for antibiotic delivery.

20 HepDirect prodrugs are cleaved via a hepatocyte-specific cytochrome P450 enzyme, CYP3A4,

21 and are resistant to cleavage by other human esterases (33). Selective bioactivation of prodrugs

22 within microbes would not only increase the circulating half-life, but may also improve the

23 therapeutic selectivity of therapeutics that target microbial enzymes with human homologs.

24 Understanding the molecular basis of host and microbe prodrug activation will facilitate design

25 of microbially targeted prodrugs. 
1 In this study, we use two zoonotic staphylococcal species, S. schleiferi and S.

2 pseudintermedius, to uncover the enzymatic mechanism of prodrug activation in staphylococci.

3 We identify and characterize the first bacterial carboxy ester prodrug activating enzyme, GloB, a

4 type II glyoxalase. Using detailed biochemical analyses, we demonstrate that GloB recognizes

5 the carboxy ester portion of the prodrug and is responsible for prodrug activation. Since GloB

6 homologues are broadly maintained, yet have substantial sequence variation, we propose that

7 this group of enzymes may be a strategy towards microbe-specific prodrug targeting.

8

A

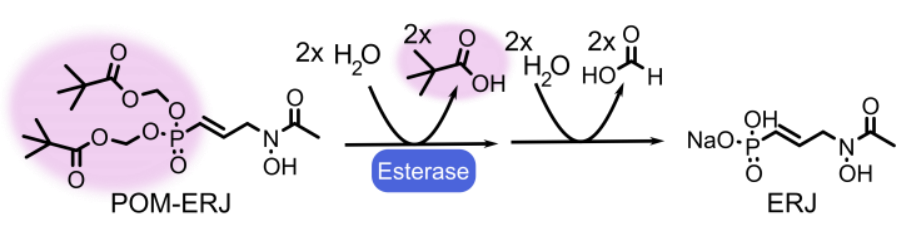

B

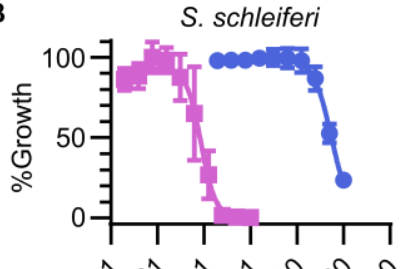

$0.0 .0 .0,0,0$

[Compound] $\mu \mathrm{M}$
S. pseudintermedius

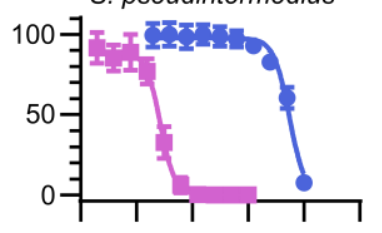

$0.0,0,0,0,0$

[Compound] $\mu \mathrm{M}$

$\longrightarrow$ ERJ - POM-ERJ

C

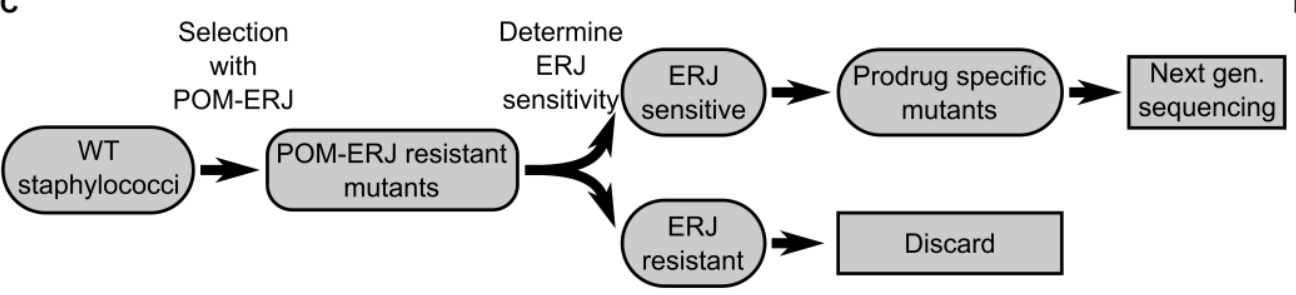

D

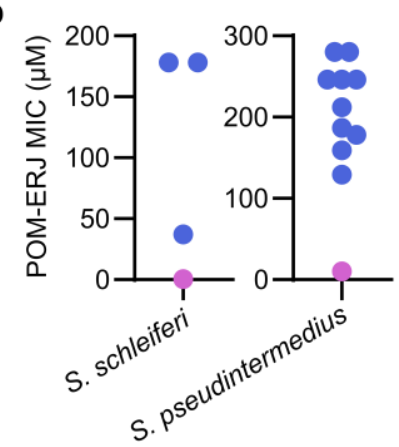

Figure 1. (A) Predicted POM-ERJ activation pathway. POM-promoiety highlighted in pink. (B) Dose-dependent growth inhibition of zoonotic staphylococci, S. schleiferi (left) and S. pseudintermedius (right), by ERJ (blue) and POM-ERJ (pink). Displayed values are the means \pm SD of three independent experiments performed in technical duplicate. (C) Screening strategy to identify prodrug activating enzymes. (D) Distribution of MIC values for WT (pink) and POM-ERJ resistant mutants from S. schleiferi (left) and S. pseudintermedius (right). Displayed values are the means values for each strain from three independent experiments performed in technical duplicate.

\section{Results}

\section{Selection of prodrug-resistant staphylococci.}

11 In our previous study, we identified phosphonate antibiotics with activity against zoonotic staphylococci (S. schleiferi and S. pseudintermedius) (27). Lipophilic carboxy ester prodrug 
1 presumably through increased cellular penetration (Fig. 1A, B). However, prodrug modifications

2 block direct engagement of inhibitors with their enzyme target (27). For this reason, we

3 hypothesized that one or more staphylococcal esterases were required for intracellular prodrug

4 activation (Fig. 1A). To identify candidate prodrug activating enzymes, we designed a genetic

5 screen/counter-screen strategy to enrich for staphylococcal strains that fail to activate lipophilic

6 ester prodrugs.

8 In our strategy, we took advantage of inhibitor pairs with the same target engagement, with and

9 without prodrug modification. We employed the phosphonate antibiotic ERJ, which selectively

10 inhibits the intracellular enzyme deoxyxylulose phosphate reductoisomerase (DXR), and POM-

11 ERJ, the bis-pivaloyloxymethyl prodrug form of ERJ, which inhibits intracellular DXR even

12 though it has been shown to lack direct activity against purified recombinant DXR in vitro (27).

13 We sought to enrich for staphylococcal strains that were resistant to prodrugged inhibitors (e.g.

14 POM-ERJ) but remained sensitive to the parent phosphonate ERJ itself (27). For this reason,

15 we first isolated staphylococcal colonies that arose from solid media containing POM-ERJ. Next,

16 we screened these POM-ERJ-resistant isolates for cross-resistance to our parent compound,

17 ERJ. POM-ERJ-resistant strains that remained sensitive to ERJ were subjected to whole

18 genome sequencing to identify candidate genetic mutations giving rise to the resistance

19 phenotype (Fig. 1C). To identify conserved resistance mechanisms, we performed this

20 screen/counter-screen independently in two staphylococcal species, S. schleiferi and S.

21 pseudintermedius. We isolated and characterized a total of 18 POM-ERJ-resistant

22 staphylococcal strains, with $\mathrm{MIC}_{90}$ values $~ 10-50$ fold higher than that of the respective wild-type

23 (WT) parental lines (Fig. 1D).

25 POM-ERJ resistance does not alter cell wall size in staphylococci. 
1 In previous work, we and others have found that cellular entry of the phosphonate antibiotic ERJ

2 and ERJ analogs requires the phosphonate transporter GIpT (16, 27, 34, 35). In contrast, entry

3 of POM-ERJ is transporter-independent $(16,27)$. POM-ERJ resistance could therefore arise

4 through cell wall modifications that directly disrupt cell penetration of prodrugs. Such cell wall

5 alterations might therefore lead to cross-resistance to other antimicrobials, such as daptomycin

6 or vancomycin. To establish the selectivity of POM-ERJ-resistance, we determined the

7 antimicrobial sensitivity of a subset of our prodrug-resistant strains against a panel of 18 clinical

8 antibiotics with diverse mechanisms-of-action. We find that POM-ERJ-resistant strains are not

9 cross-resistant to other inhibitors, including daptomycin and vancomycin (Table S1), suggesting

10 a prodrug-specific mechanism of resistance. Additionally, we quantified the cell wall size in

11 POM-ERJ-resistant staphylococci by transmission electron microscopy, because an established daptomycin and vancomycin resistance strategy for $S$. aureus is the generation of thickened cell
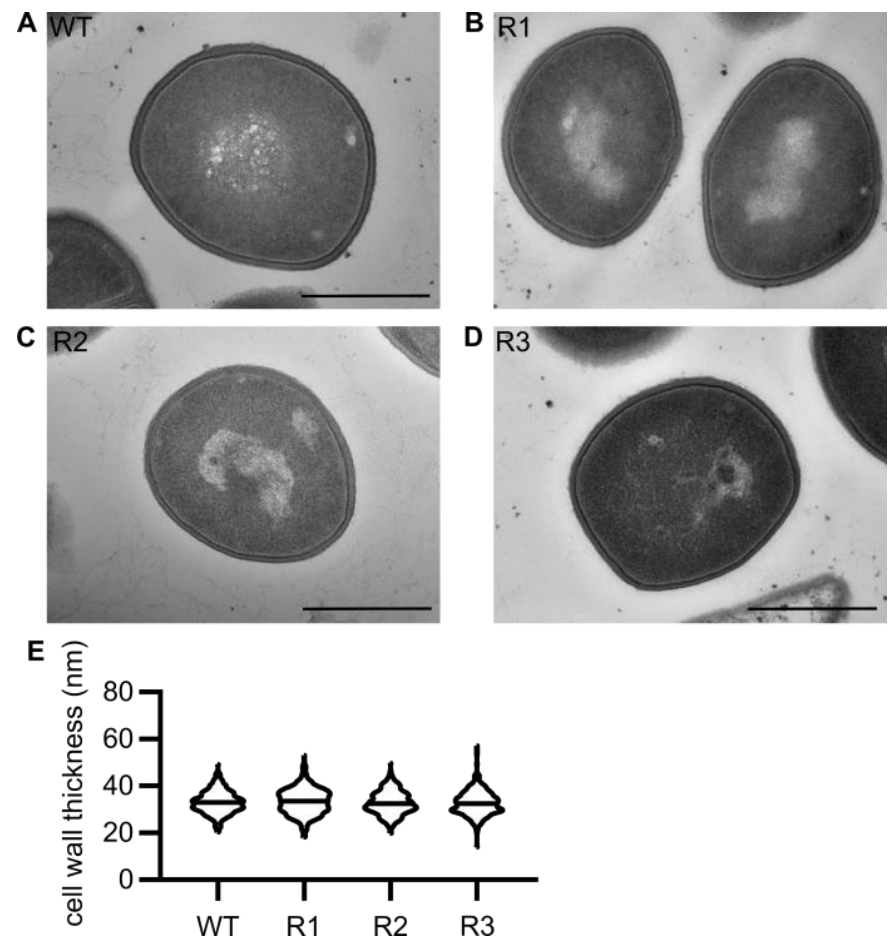

Figure 2. (A-D) Representative transmission electron micrographs of WT (A) or three independent POM-ERJ resistant $S$. schleiferi strains (B-D). Scale bars $=500 \mathrm{~nm}$. (E) Distribution of cell wall thickness in WT and POM-ERJ resistant $S$. schleiferi as measured in a total of 300 cells from three independent experiments of 100 cells each. Midline indicates mean of all measurements. 
1 walls that reduce inhibitor entry $(36,37)$. We find no changes in cell wall thickness in prodrug-

2 resistant isolates compared to their prodrug-sensitive WT parental lines (Fig 2).

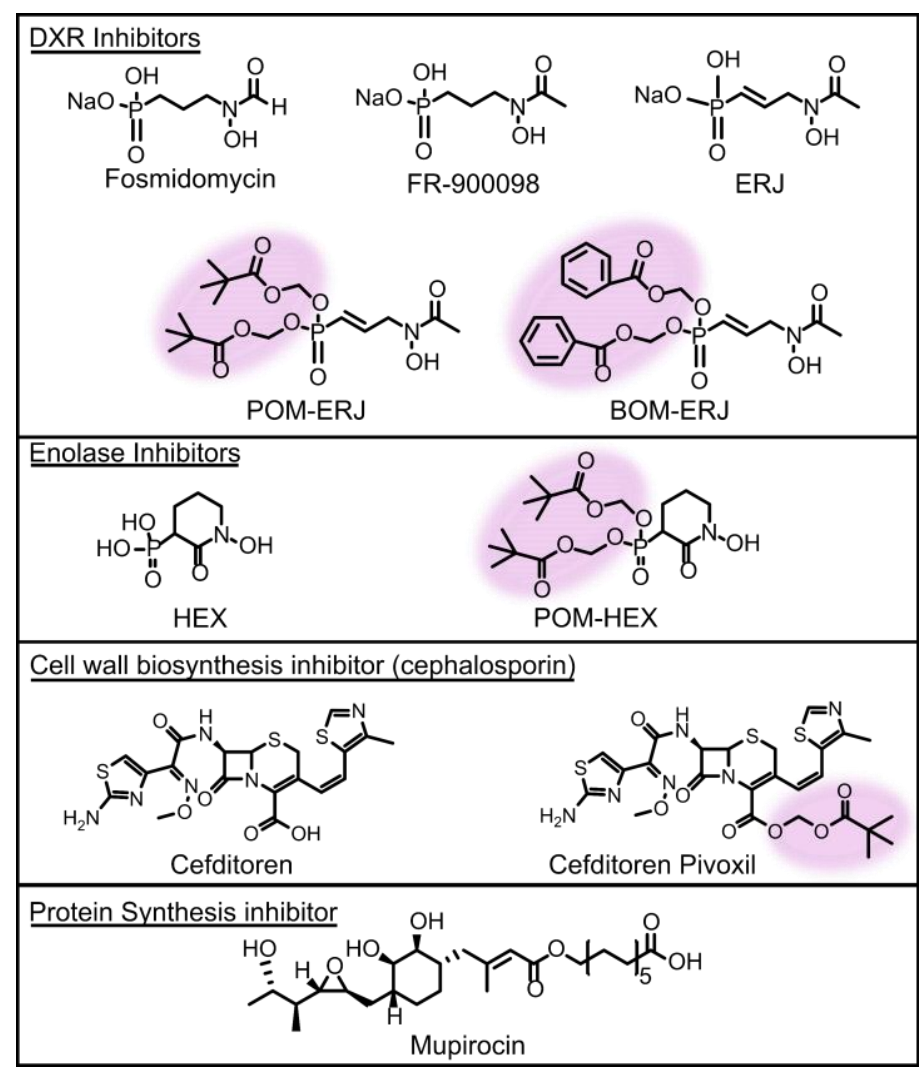

Figure 3. Structures of antistaphylococcal inhibitors used in this study. Structures are grouped by mechanism of action. For prodrugged compounds, promoieties are highlighted in pink.

\section{POM-ERJ-resistant staphylococci are cross-resistant to other carboxy ester prodrug}

\section{5 antibiotics.}

6 If POM-ERJ resistance is due to loss of a prodrug activating enzyme(s), we hypothesized that

7 POM-ERJ-resistant staphylococci would likewise be cross-resistant to other carboxy ester

8 prodrug antibiotics. To evaluate this possibility, we selected several additional pairs of inhibitors

9 (carboxy ester prodrugs and their cognate parent (non-prodrugged) compounds), with distinct

10 cellular targets (e.g. penicillin binding protein, deoxyxylulose reductoisomerase (DXR), and 


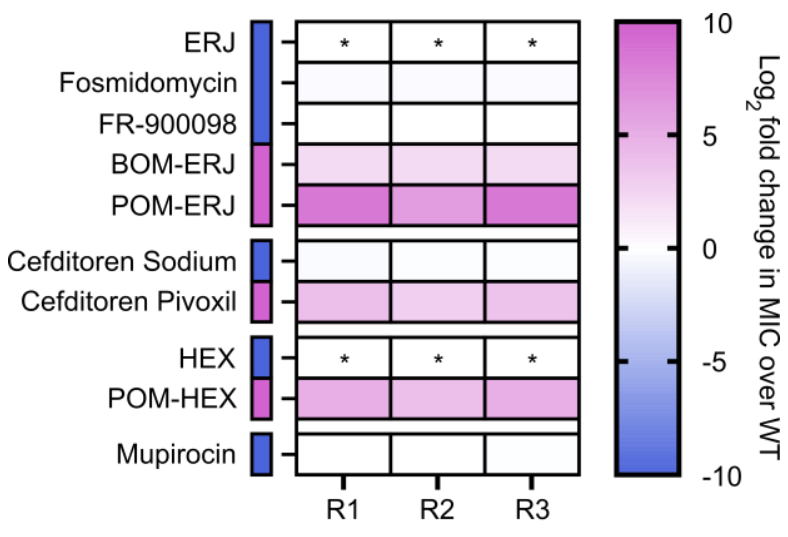

Non-prodrug $\square$ Carboxyester prodrug

Figure 4. Cross-resistance to lipophilic ester prodrugs in POM-ERJ-resistant S. schleiferi. WT and POM-ERJ resistant $S$. schleiferi were treated with the compounds displayed in Figure 3. Compounds are grouped by mechanism of action and color coded to indicate whether a given compound is a carboxy ester prodrug. Displayed are the mean values of the fold change (resistant isolate/WT) of three independent experiments performed in technical duplicate. * indicates compounds whose MIC values were too high to measure. Numerical data additionally provided in Table S2.

enolase) (Fig. 3) (22, 32). For three of our POM-ERJ-resistant S. schleiferi isolates, we determined the minimum inhibitory concentration (MIC) for each compound, compared to the WT parental strain (Fig. 4).

We find that POM-ERJ-resistant staphylococci remain equally sensitive to non-prodrugged compounds (such as ERJ analogues) and the third-generation cephalosporin cefditoren. In contrast, POM-ERJ-resistant staphylococci exhibit significantly increased MICs to multiple classes of lipophilic ester prodrugs, exhibiting cross-resistance to both cefditoren pivoxil (cell

9 wall inhibitor) and POM-HEX (inhibitor of enolase) (Fig. 4, Table S2). Thus, POM-ERJ-resistant

10 staphylococci are cross-resistant to other POM-prodrug inhibitors, regardless of the intracellular

11 target. Our data suggest that POM-prodrugs follow a common and conserved activation

12 mechanism that has been disrupted in our POM-ERJ-resistant isolates. 
1 To explore how changes in the chemical structure of the prodrug group impacts prodrug

2 resistance, we also evaluated whether our POM-ERJ-resistant isolates were cross-resistant to

3 antimicrobial prodrugs that possess another common carboxy ester prodrug moiety,

4 benzyloxymethyl (BOM) (Fig. 3). Indeed, we find our POM-ERJ-resistant isolates are also cross-

5 resistant to BOM-ERJ (Fig. 4).

6

7 Carboxy ester prodrugs are more lipophilic than their parental molecules. To evaluate whether

8 prodrug resistance in our strains is driven by the lipophobicity of the molecule rather than its

9 ester bond, we selected an additional highly lipophilic antibiotic, mupirocin, which inhibits protein

10 biosynthesis (Fig. 3). POM-ERJ-resistant staphylococci were not cross-resistant to mupirocin,

11 further supporting that prodrug resistance in these strains is specific to the carboxy ester bond

12 of the prodrug (Fig. 4).

POM-ERJ resistant staphylococci are enriched in mutations in the GloB gene.

15 To characterize the genetic changes associated with carboxy ester prodrug resistance, we

16 performed whole genome sequencing of prodrug resistant isolates of both $S$. schleiferi and $S$.

17 pseudintermedius. The whole genomes of each isolate were compared to the respective

18 parental genome and candidate genetic changes were verified by Sanger sequencing. We

19 prioritized nonsynonymous genetic changes that were represented in more than one strain. A

20 complete list of identified mutations is found in Table S3.

22 In both independent genetic screens, we found that prodrug resistant staphylococci were

23 enriched in mutations in an evolutionarily conserved locus. We identified multiple isolates (3/16

24 S. schleiferi, 14/18 S. pseudintermedius) with sequence modifications in the locus annotated as

25 hydroxyacylglutathione hydrolase, gloB (LH95_06060 in S. schleiferi, SPSE_1252 in S.

26 pseudintermedius, Table S3). Most genetic changes in gloB were nonsynonymous single 


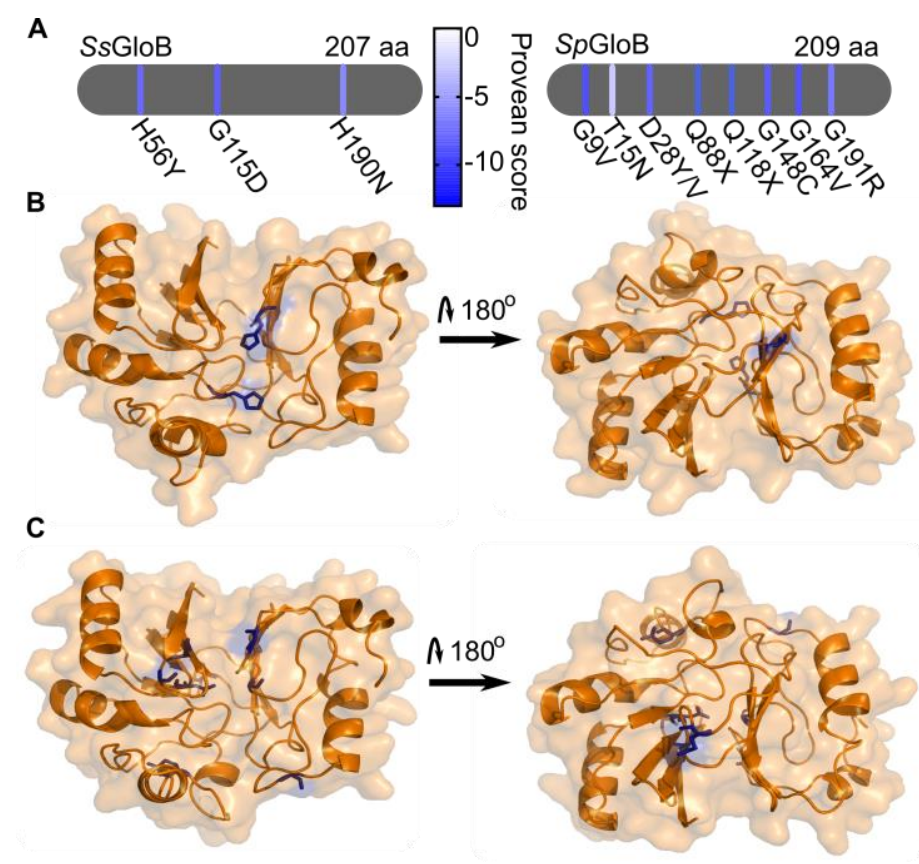

Figure 5. POM-ERJ resistant staphylococci are enriched for mutations in the locus encoding hydroxyacylglutathione hydrolase (GloB). (A) Locations and identities of GloB mutations discovered by whole-genome sequencing and independently verified by Sanger sequencing. Line coloring represents predicted impact of a given mutation on GloB function, scores below -2.5 are predicted to be deleterious. $(\mathrm{B}, \mathrm{C})$ Homology models of $S$. schleiferi $(\mathrm{B})$ and S. pseudintermedius GloB generated using SWISSMODEL. Residues found to be mutated in POM-ERJ resistant staphylococci explicitly shown in blue.

nucleotide polymorphisms, though two nonsense alleles that would truncate approximately $50 \%$ of the protein were also identified (Fig. 5, Table S3). In several strains, the only genetic variation

3 that distinguished WT and resistant genomes was within the gloB locus.

5 Of the 17 identified GloB mutations, 12 unique alleles were identified in prodrug-resistant

6 staphylococci. Using PROVEAN, an algorithm which quantifies the predicted impact of amino

7 acid substitutions on protein function, each of these 12 alleles is predicted to have deleterious

8 effects on protein function (below the threshold score of -2.5) (Fig. 5) (38). S. schleiferi and S.

9 pseudintermedius are non-model organisms that possess endogenous CRISPR-Cas9 systems

10 and transformation of these organisms has not yet been described (39). Attempts to ectopically

11 complement gloB mutant strains with WT GloB (>90 independent transformation attempts using 
1 established methods for $S$. aureus, S. epidermidis, and B. subtilis) were unsuccessful in

2 recovering transformed colonies, despite preparing plasmid from the $S$. aureus restriction

3 deficient cloning intermediate, RN4220, and the cytosine methyltransferase negative E. coli

4 mutant, DC10B (40-46). However, the independent selection of 12 unique loss-of-function

5 alleles in two different species strongly suggests that loss of GloB function is responsible for

6 prodrug resistance in S. schleiferi and S. pseudintermedius.

8 Structural basis of GloB loss-of-function.

9 As prodrug-resistance mutations in GloB map along its entire linear sequence, we next

10 examined the structural basis for GloB loss-of-function. We generated homology models of both

11 SsGloB and SpGloB using SWISS-MODEL (47). The resulting staphylococcal model is based

12 on the sequence-similar metallo- $\beta$-lactamase superfamily member from Thermus thermophilus

13 (PDB 2ZWR) (48). This hit had a global model quality estimate (GNQE) of 0.71 and 0.70 for $S$.

14 schleiferi and S. pseudintermedius GloB homologs, respectively, suggesting the built models

15 are reliable and accurate. In both protein models, we find that POM-ERJ-resistance mutations

16 are primarily located towards the interior of the protein, occupying the same cavity as the well

17 conserved glyoxalase II metal binding motif $(\mathrm{THxHxDH})(49)$. This modeling thus indicates that

18 these prodrug-resistance alleles impair the GloB active site (Fig. 5).

20 GloB is a functioning type II glyoxalase, not a $\beta$-lactamase.

21 GloB is predicted to be a type II glyoxalase and a member of the large metallo- $\beta$-lactamase

22 protein superfamily (INTERPRO IPR001279). Members of this superfamily hydrolyze thioester, 23 sulfuric ester, and phosphodiester bonds, such as the ester linkage present in POM-ERJ (49-

24 52). Type II glyoxalases catalyze the second step in the glyoxalase pathway that is responsible

25 for the conversion of methylglyoxal (a toxic byproduct endogenously produced during 
1 metabolism) to lactic acid. Specifically, GloB catalyzes the conversion of D-lactoylglutathione to D-lactate.

\section{A}

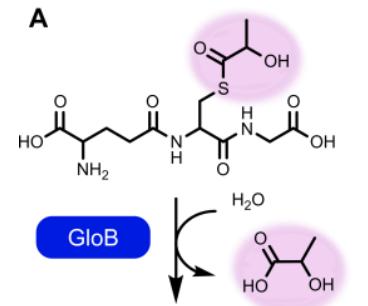

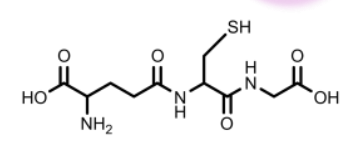

$\mathcal{C}_{\text {TNB }}^{\text {DTNB }}\left(\begin{array}{c}\text { Abs } \\ 412 \mathrm{~nm}\end{array}\right)$

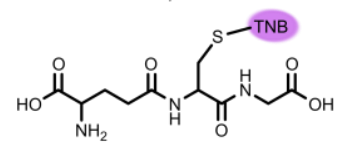

B

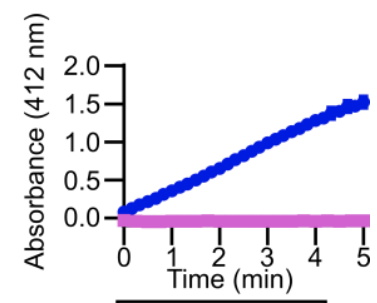

- SsGloB

$\because S s G l o B$ H54N

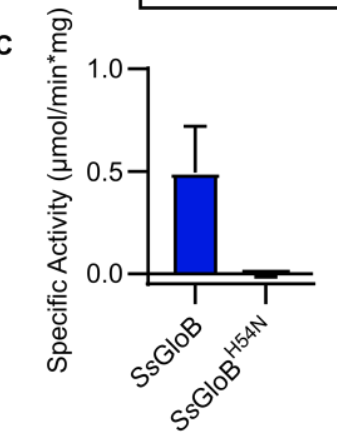

Figure 6. (A) Enzymatic catalysis of S-lactoylglutathione by GloB. DTNB conversion to TNB results in increased absorbance at $412 \mathrm{~nm}$. (B) Reaction progress curve for SsGloB (blue) and catalytically inactive SsGloB H54N (pink), using S-lactoylglutathione as a substrate. (C) SsGloB and SsGloB H54N specific activity for S-lactoylglutathione. Displayed are the means \pm SD from three independent experiments preformed in technical duplicate.

4 To determine whether SsGloB encodes a functional type II glyoxalase, we evaluated whether

5 SsGloB hydrolyzes S-lactoylglutathione using an assay in which hydrolysis of S-

6 lactoylglutathione is linked to a change in absorbance (Fig. 6A). We purified recombinant WT

7 SsGloB protein and its catalytically inactive variant, SsGloB ${ }^{\mathrm{H} 54 \mathrm{~N}}$, in which the histidine of the

8 canonical metal binding motif $(\mathrm{TH} x \mathrm{H} \times \mathrm{DH})$ has been altered to an asparagine (Fig. S1) $(49,51$,

9 52). We find that $S s G l o B$, but not $S s G{ }^{2} B^{H 54 N}$, hydrolyzes S-lactoylglutathione with a specific

10 activity of $0.493 \mu \mathrm{mol}^{*} \mathrm{~min}^{-1} \mathrm{mg}^{-1}$ (Fig. S2, Fig. 6B,C). This activity is similar to other

11 characterized microbial type II glyoxalases (Saccharomyces cerevisiae, $1.34 \mu \mathrm{mol}^{*} \mathrm{~min}^{-1} \mathrm{mg}^{-1}$;

12 Trypanosoma brucei, $\sim 8 \mu \mathrm{mol}^{*} \mathrm{~min}^{-1} \mathrm{mg}^{-1}$ ), but is much lower than that of previously

13 characterized type II glyoxalases from plants and mammals $\left(20-2000 \mu \mathrm{mol}^{\star} \mathrm{min}^{-1} \mathrm{mg}^{-1}\right)(53-61)$. 
1 We determined the metal dependence of SsGloB and find that SsGloB is a functional type II

2 glyoxalase in manganese, cobalt, calcium, and zinc, with a modest preference noted towards

3 magnesium (Fig. S3).

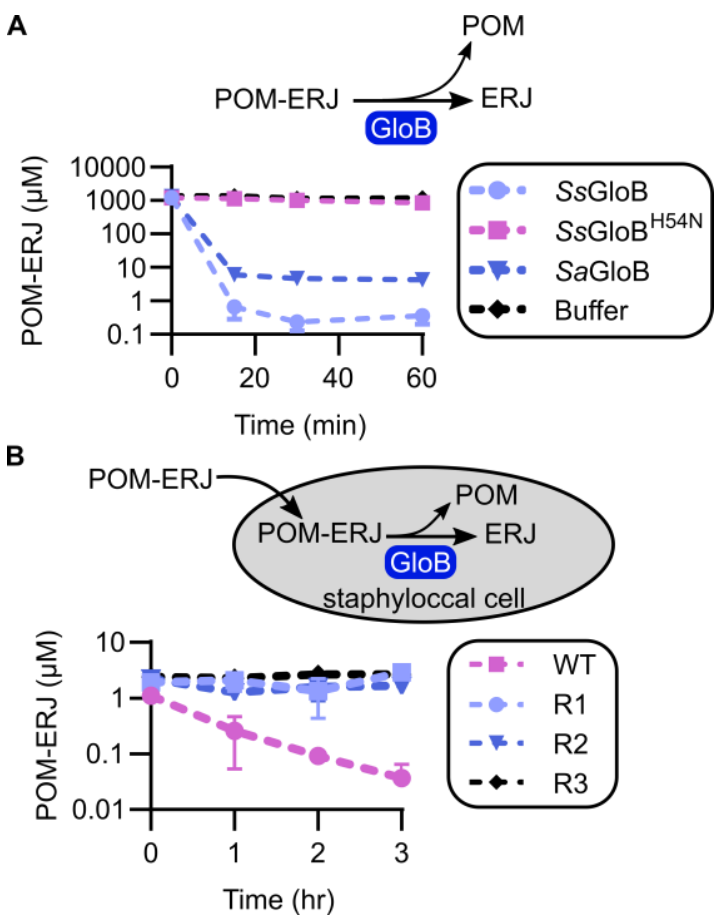

Figure 7. (A) Recombinant SsGloB, catalytically inactive SsGloB H54N, GloB from S. aureus (SaGloB), or buffer were incubated with POM-ERJ and prodrug concentrations were measured by LC-MS. (B) Wild-type and POM-ERJ-resistant gloB mutant $S$. schleiferi isolates were treated with POM-ERJ and intracellular drug concentrations were measured by LC-MS. Displayed are the mean values \pm SD from three independent experiments. Error bars may not be visible due to precision in measurement.

5 As some members of the metallo- $\beta$-lactamase protein superfamily mediate hydrolysis of $\beta$ -

6 lactam antibiotics, we considered whether GloB also had $\beta$-lactamase activity. Because gloB

7 mutant strains are not cross-resistant to the $\beta$-lactam-containing antibiotics (except for the

8 prodrugged cephalosporin, cefditoren pivoxil) (Fig. 4, Table S2), we predicted that GloB was not

9 a functional metallo- $\beta$-lactamase. As expected, we find that SsGloB does not hydrolyze the $\beta$ -

10 lactamase ring of nitrocefin (a canonical $\beta$-lactamase substrate), in contrast to the active $B$.

11 cereus $\beta$-lactamase (Fig. S2). 
Staphylococcal GloB hydrolyzes POM-ERJ in vitro and in vivo.

2 Loss-of-function mutation in GloB is associated with resistance not only to POM-ERJ, but also

3 to other ester prodrugs. Because GloB does not mediate resistance to ERJ or other

4 phosphonates, our data suggested that GloB might directly catalyze the conversion of POM-

5 ERJ to ERJ. To determine whether GloB de-esterifies POM-ERJ, we developed a liquid

6 chromatography-mass spectrometry (LC-MS)-based assay to quantify POM-ERJ

7 concentrations. Incubation of purified recombinant SsGloB protein, but not its inactive variant

$8\left(\right.$ SsGloB $\left.{ }^{\mathrm{H} 54 \mathrm{~N}}\right)$, with POM-ERJ results in rapid loss of POM-ERJ, consistent with SsGloB-

9 mediated cleavage (Fig. 7A). To determine whether prodrug activation activity is conserved

10 among staphylococcal GloB homologs, we also purified recombinant GloB from the human

11 pathogen S. aureus (Fig. S1). We find that SaGloB also directly hydrolyzes POM-ERJ (Fig. 7A).

13 To determine whether GloB mediates intracellular prodrug activation, we evaluated the

14 intracellular concentrations of POM-ERJ in drug-treated WT and gloB mutant staphylococci. We

15 prepared staphylococcal cultures treated with POM-ERJ and quenched the reaction at several

16 timepoints to monitor the course of intracellular prodrug depletion. As expected, we find that

17 POM-ERJ is rapidly depleted in WT S. schleiferi, consistent with enzymatic activation. In

18 contrast, POM-ERJ concentrations do not decrease over time in gloB mutant strains, in which

19 the sole genetic change in each strain compared to WT is in the gloB locus (Fig. 7B). This

20 suggests that the initial step in carboxy ester prodrug activation in staphylococci lacks functional

21 redundancy and is exclusively dependent on GloB.

\section{Staphylococcal GloB enzymes represent a distinct clade of bacterial glyoxalases.}

25 Because staphylococcal GloB mediates de-esterification of ester prodrugs, we sought to 26 evaluate the feasibility of using these enzymes to design prodrugs specifically targeted for 
1 activation in staphylococci. We constructed a phylogenetic tree of GloB homologs across

2 diverse microbial genomes, as well as in humans and mice (Fig. S4A), specifically including

3 sequences of previously characterized GloB homologs. We find that considerable sequence

4 variation exists within GloB homologs, with no clear clustering by phylogeny except for those

5 GloB homologs originating in plants and mammals. This contrasts with a phylogenetic tree

6 generated using the DNA-directed RNA polymerase subunit beta (rpoB), which generally follows

7 the traditional tree of life (Fig. S4B).

9 While sequence differences between staphylococcal GloB and human GloB suggest that there

10 may be substrate utilization differences between humans and staphylococci, ultimately

11 differences within the active site are likely to drive substrate specificity. Using pymol, we aligned

12 our homology model of SsGloB with the glutathione bound GloB from humans (PDB ID: 1qh5)

$13(62,63)$. The two structures align well with a root-mean-square deviation (RMSD) of $1.528 \AA$,

14 and are well conserved in the overall structure as well as the characteristic $\mathrm{Zn}$ binding motif,

$15 \mathrm{THxHxDH}$ (Fig. S5A,B). Notably, however, HsGloB has a significant C-terminal extension which

16 is not present in SsGloB. This C-terminal extension forms an $\alpha$-helix which borders the active

17 site and contains two residues, K252 and R249, which appear to be involved in coordinating the

18 co-crystallized glutathione substrate (Fig. S5C). The absence of this C-terminal extension in our

19 SsGloB homology model suggests that HsGloB and SsGloB have distinct active site chemistry

20 that may be exploited to drive prodrug activation selectively by SsGloB vs $H s G l o B$.

\section{DISCUSSION}

23 Antimicrobial resistance is a substantial challenge for treatment of both human and animal

24 staphylococcal infections. Widespread methicillin resistance contributes both to poor clinical 
1 outcomes and increased treatment costs, and resistance is emerging to agents of last resort

2 such as vancomycin and linezolid (7). Current antimicrobial therapies target a fraction of

3 essential cellular processes, and metabolism remains a promising area for therapeutic

4 development $(11,12)$. Many metabolic genes are essential for growth, especially in the nutrient

5 limited setting of infection (64-67). Additionally, chemical ligands are readily designed with high

6 potency by mimicking natural substrates used by metabolic enzymes. Finally, because active

7 site mutations that disrupt binding of competitive inhibitors are likely to deleteriously affect

8 enzyme function, the barrier to resistance can be high $(68,69)$. Although many metabolic

9 processes are conserved between humans and microbes, selective targeting of microbes is

10 achievable as is demonstrated by the success of folate antagonists

11 (trimethoprim/sulfamethoxazole) and bedaquiline $\left(a F_{0} F_{1}\right.$ ATP synthase inhibitor of

Mycobacterium tuberculosis) (70-72).

14 Unfortunately, many metabolic inhibitors require cell-impermeable phosphonic acids for efficient 15 target inhibition. Prodrugging strategies to increase cellular penetration have been developed 16 for a variety of therapeutics, most notably the anti-cancer and anti-viral nucleosides (19). These

17 prodrug strategies must be labile-enough that the compound is activated within the target cell,

18 yet stable enough to resist premature prodrug activation by the sera. Prodrugs which are

19 selectively activated within target cells have the added benefit of reducing off-target toxicity

20 effects. To achieve cell-targeted prodrug activation, knowledge of the activation mechanisms in

21 sera, as well the target cell, are essential. While prodrug targeting has been achieved for liver

22 therapies, this strategy has yet to be employed for bacterial antibiotics that employ ester

23 prodrug moieties (33).

25 In this work, we have identified a new mechanism for the de-esterification and activation of

26 lipophilic ester prodrugs though a conserved staphylococcal esterase in the metallo- $\beta$ - 
1 lactamase superfamily. Loss-of-function of GloB confers resistance to lipophilic carboxy ester

2 prodrugs in two zoonotic pathogens, S. schleiferi and S. pseudintermedius (Fig. 1D, Table S3).

3 Purified recombinant GloB from S. schleiferi and the related human pathogen S. aureus directly

4 catalyzes pro-drug de-esterification in vitro (Fig. 7A). Because gloB mutant staphylococci are

5 cross-resistant to other POM-containing prodrugs that differ in "warhead" and intracellular

6 targets (Fig. 4), we propose that substrate-specificity of GloB appears driven by recognition of

7 the lipophilic promoiety, rather than the target inhibitory portion of each compound.

8

9 Bacterial prodrug ester activation through GloB hijacks a conserved bacterial protective

10 mechanism in bacteria, as hydroxyacylglutathione hydrolase represents the second enzyme of

11 the two-step glyoxalase pathway. During normal metabolism, the glycolytic intermediates

12 glyceraldehyde-3-phosphate (GAP) and dihydroxyacetone phosphate (DHAP) undergo

13 nonenzymatic decomposition to methylglyoxal, a toxic metabolite. GloB is required for

14 methylglyoxal detoxification, as methylglyoxal is highly reactive and irreversibly glycates

15 proteins and nucleic acids (73-75). In S. aureus, methylglyoxal accumulation potentiates

16 antibiotic susceptibility (76). In addition, methylglyoxal is itself directly antibacterial and

17 postulated to be the primary antistaphylococcal ingredient in Manduka honey (used on chronic

18 wounds) (76-79). Our studies suggest that strains of S. schleiferi and S. pseudintermedius

19 lacking GloB have preserved axenic growth in rich media, which raises concern for the ease of

20 resistance development when GloB-targeted prodrugs are used as anti-infectives. However, the

21 known toxicity of methylglyoxal in a host infection setting suggests that reduced methylglyoxal

22 detoxification as the result of GloB loss-of-function would not be well tolerated in vivo.

24 Identification of GloB as a prodrug activating enzyme in staphylococci is a major step forward

25 for highly selective microbial targeting of compounds. Though GloB homologs are widespread in 26 microbes and are present in humans, significant sequence variation exists in GloB sequences, 
1 which results in a variety of GloB substrate preferences (Fig. S4). For example, human GloB

2 has an additional $\alpha$-helix along the active site that introduces two additional residues, K252 and

3 R249 to the substrate binding pocket (Fig. S5) (63). These residues, and this $\alpha$-helix, are

4 notably absent in microbial GloBs, suggesting that there are underlying substrate differences

5 between human and microbial GloB enzymes. Furthermore, there is substantial sequence

6 variation in GloB orthologs across all microbes, suggesting that GloB substrate specificities may

7 discern between individual clades of bacteria. We expect that development of prodrugs specific

8 to GloB would result in a narrow-spectrum antibiotic which would reduce off-target effects on the

9 microbiome and decrease the broad pressure to evolve resistance.

11 Materials and Methods

12 Inhibitors. Fosmidomycin (Millipore Sigma) and FR-900098 (Millipore Sigma) were

13 resuspended in sterile water. POM-ERJ and POM-HEX were synthesized and stored in DMSO

14 as described (29, 32). Cefditoren pivoxil (Millipore Sigma), cefditoren sodium (Clearsynth), and

15 mupirocin (Millipore Sigma) were resuspended in DMSO. The synthesis of BOM-ERJ will be 16 reported elsewhere.

Generation of POM-ERJ-resistant mutants in S. schleiferi and S. pseudintermedius.

19 Clinical isolates of S. schleiferi (S53022327s) and S. pseudintermedius (H20421242p) were

20 cloned and adapted to laboratory media through three rounds of sequential colony isolation and

21 growth on Luria Broth (LB) agar plates. The isolated POM-ERJ-sensitive parental clones were

22 incubated overnight on LB agar containing POM-ERJ at $3.56 \mu \mathrm{M}$ and $7.12 \mu \mathrm{M}$ for S. schleiferi

23 and $11.2 \mu \mathrm{M}$ and $22.4 \mu \mathrm{M}$ for $S$. pseudintermedius. Surviving single colonies were re-struck

24 onto LB agar for clonal isolation. POM-ERJ resistance of isolated clones was confirmed by 
overnight growth on LB agar containing POM-ERJ (3.56-22.4 $\mu \mathrm{M})$. The POM-ERJ-sensitive parental clones were used as a control to confirm growth and antibiotic resistance.

4 Quantification of resistance. Minimum Inhibitory Concentration (MIC) assays were performed

5 using microtiter broth dilution in clear 96-well plates (80). Compounds were serially diluted in

6 duplicate for a total of 10 serial dilutions. Top well concentrations were: POM-ERJ $280 \mu \mathrm{M}$,

7 BOM-ERJ $53.95 \mu \mathrm{M}, \mathrm{KMH}-10253.95 \mu \mathrm{M}$, cefditoren pivoxil $201.38 \mu \mathrm{M}$, cefditoren sodium 56.65

$8 \mu \mathrm{M}, \mathrm{POM}-\mathrm{HEX} 100 \mu \mathrm{M}$, mupirocin $2.50 \mu \mathrm{M}$, FR-900098 $1 \mathrm{mM}$, fosmidomycin $100 \mu \mathrm{M}$. Bacteria

9 cultured without drug were used as a positive control for growth, and LB without bacteria was

10 used as a negative control for contamination. Plates were inoculated with $75 \mu \mathrm{L}$ bacteria diluted

11 to $1 \times 10^{5} \mathrm{CFU} / \mathrm{mL}$ in LB. After inoculation, plates were incubated for $16-24 \mathrm{~h}$ while shaking at

$12200 \mathrm{RPM}$ at $37^{\circ} \mathrm{C}$. Plates were visually inspected, and the lowest concentration of antibiotic

13 suppressing visual growth was recorded as the MIC. All experiments were performed at least in

14 triplicate and data reported represent the mean \pm SD.

Transmission Electron Microscopy. For ultrastructural analysis, bacteria were cultured in 5

$17 \mathrm{~mL}$ LB while shaking at $37^{\circ} \mathrm{C}$ until $\mathrm{OD}_{600}=0.25-1.0 . \mathrm{A} 1 \mathrm{~mL}$ sample of exponential phase

18 bacteria was pelleted at $6,000 \mathrm{rcf}$ and resuspended in $1 \mathrm{~mL}$ fix (2\% paraformaldehyde/2.5\%

19 glutaraldehyde (Polysciences Inc., Warrington, PA) in 100 mM sodium cacodylate buffer, pH

20 7.2) for $1 \mathrm{~h}$ while rocking at RT. The fixed suspension of bacteria was washed in sodium

21 cacodylate buffer and postfixed in 1\% osmium tetroxide (Polysciences Inc.) for $1 \mathrm{~h}$. Samples

22 were then rinsed extensively in $\mathrm{dH}_{2} \mathrm{O}$ prior to en bloc staining with $1 \%$ aqueous uranyl acetate

23 (Ted Pella Inc., Redding, CA) for 1 h. Following several rinses in $\mathrm{dH}_{2} \mathrm{O}$, samples were

24 dehydrated in a graded series of ethanol and embedded in Eponate 12 resin (Ted Pella Inc.).

25 Sections of $95 \mathrm{~nm}$ were cut with a Leica Ultracut UC7 ultramicrotome (Leica Microsystems Inc.,

26 Bannockburn, IL), and stained with uranyl acetate and lead citrate. Samples were viewed at 
30,000X on a JEOL 1200EX transmission electron microscope (JEOL USA, Peabody, MA)

2 equipped with an AMT 8 megapixel digital camera (Advanced Microscopy Techniques, Woburn,

3 MA). Cell wall thickness was measured (ImageJ 1.38g customized for AMT images) for 100

4 bacteria in three independent samples (total $n=300$ ).

6 Whole genome sequencing and variant discovery. Using a standard phenol-chloroform

7 extraction and ethanol precipitation protocol, genomic DNA was isolated from overnight cultures

8 of S. pseudintermedius and S. schleiferi. Sequencing libraries were prepared and sequenced by

9 the Washington University Genome Technology Access Center (GTAC). $1 \mu \mathrm{g}$ of DNA was

10 sonicated to an average size of $175 \mathrm{bp}$. Fragments were blunt ended and had an A base added

11 to the $3^{\prime}$ end. Sequence adapters were ligated to the ends and the sequence tags were added

12 via amplification. Resulting libraries were sequenced on an Illumina HiSeq 2500 to generate 101

13 bp paired end reads. DNA quantity and quality were assessed by GTAC using Agilent

14 Tapestation.

15 For the analysis, sequences from GenBank were retrieved from the following organisms:

16 S. pseudintermedius ED99 (accession number CP002478) and S. schleiferi 1360-13

17 (CP009470) assemblies were downloaded from NCBI (ftp://ftp.ncbi.nlm.nih.gov). Paired-end

18 reads were aligned to each of the available genomes using Novoalign v3.03. (Novocraft

19 Technologies). Duplicates were removed and variants were called using SAMtools (81). SNPs

20 were filtered against parent variants and by both mean depth value and quality score (minDP

$21=5, \min Q=30)(82)$. Genetic variants were annotated using SnpEff v4.3 (83). For all samples,

22 at least $90 \%$ of the genome was sequenced at $20 x$ coverage. Whole genome sequencing data

23 is available in the NCBI BioProject database and Sequence Read Archive under the BioProject

24 ID 648133. 
Sanger sequencing of S. schleiferi and S. pseudintermedius variants. The SNPs, the reference sequences, and gene specific primers can be found in Table S4 for both S. schleiferi and S. pseudintermedius. Amplicons were sequenced by GENEWIZ.

Staphylococcal GIoB homology modeling. SWISS-MODEL (https://swissmodel.expasy.org/) was used to generate homology models. Modeling parameters were left at default. Both SsGloB and SpGloB models were built using the solved Metallo- $\beta$-lactamase superfamily protein, 2ZWR.1.A, which is $39.2 \%$ identical in sequence.

Recombinant expression and purification of GloB. WT GloB from S. schleiferi was amplified using the forward and reverse primers in Table S4. The PCR product was then cloned into the BG1861 vector by ligation-independent cloning to introduce a N-terminal 6xHis tag and transformed into Stellar ${ }^{\mathrm{TM}}$ chemically competent cells (Clontech Laboratories) for plasmid propagation (84). Proper insertion was verified using restriction digest and Sanger sequencing. For S. schleiferi protein expression, the plasmid was transformed into E. coli Arctic Express (Agilent). Cells were grown to $\mathrm{OD}_{600}=0.4-0.7$, chilled to $8^{\circ} \mathrm{C}$, and $\mathrm{GloB}$ expression was induced with $0.5 \mathrm{mM}$ isopropyl- $\beta$-D-thiogalactoside (IPTG) overnight. For $S$. aureus protein expression, the plasmid was transformed into E. coli BL21 (DE3) pLysS cells (Promega). Cells were grown to $\mathrm{OD}_{600}=0.4-0.7$ and GloB expression was induced with $0.5 \mathrm{mM}$ IPTG for $2 \mathrm{~h}$. Cells were harvested by centrifugation at $4274 \times \mathrm{g}$ for $5 \mathrm{~min}$ at $4^{\circ} \mathrm{C}$. The cell pellet was lysed by sonication

21 in $50 \mathrm{~mL}$ lysis buffer containing $25 \mathrm{mM}$ Tris $\mathrm{HCl}(\mathrm{pH}$ 7.5), $20 \mathrm{mM}$ imidazole, $1 \mathrm{mM} \mathrm{MgCl}, 1 \mathrm{mM}$ 22 dithiothreitol (DTT), $1 \mathrm{mg} / \mathrm{mL}$ lysozyme, $75 \mathrm{U}$ benzonase and 1 Complete Mini EDTA-free 23 protease inhibitor tablet (Roche Applied Science). Insoluble proteins were removed by

24 centrifugation twice at $20,000 \times \mathrm{g}$ for $20 \mathrm{~min}$ each. The hexahistidine-tagged DXR protein was

25 affinity purified from soluble lysate via nickel agarose beads (Gold Biotechnology). Bound 26 protein was eluted in $300 \mathrm{mM}$ imidazole, $25 \mathrm{mM}$ Tris $\mathrm{HCl}(\mathrm{pH} 7.5), 1 \mathrm{mM} \mathrm{MgCl}, 10 \%$ glycerol, 
1 and $250 \mathrm{mM} \mathrm{NaCl}$. Affinity purified protein was further purified over a HiLoad 16/60 Superdex

2200 gel filtration column (GE Healthsciences) using an AKTAExplorer 100 FPLC (GE

3 Healthsciences). FPLC buffer contained $25 \mathrm{mM}$ Tris $\mathrm{HCl}(\mathrm{pH} 7.5), 250 \mathrm{mM} \mathrm{NaCl}, 1 \mathrm{mM} \mathrm{MgCl} 2$

4 and $10 \%$ glycerol. Fractions containing $>90 \%$ pure enzyme (evaluated by SDS-PAGE) were

5 concentrated by centrifugation using Amicon Ultra-15 centrifugal filter units (EMD Millipore) and

6 flash frozen in liquid nitrogen before permanent storage at $-80^{\circ} \mathrm{C}$. Protein identity was verified

7 using mass spectrometry at the University of Nebraska.

8

9 GloB mutant generation. WT GloB for S. schleiferi was synthesized by GeneWiz, Inc (Beijing,

10 China) with a CAT->AAT mutation in the 54th codon (H54N) and cloned into the BG1861 vector

11 to introduce an N-terminal 6xHis tag. Proper insertion was verified by Sanger sequencing.

Glyoxalase II activity assay. S. schleiferi GloB was tested for type II Glyoxalase activity as

14 previously with minor changes (50). $50 \mu \mathrm{L}$ reactions containing $25 \mathrm{mM}$ Tris pH 7.5, $250 \mathrm{mM}$

$15 \mathrm{NaCl}, 1 \mathrm{mM}$ divalent salt, 10\% glycerol, 200 MM 5,5'-Dithiobis(2-nitrobenzoic acid) (DTNB,

16 Sigma D8130), and $1 \mathrm{mM}$ D-lactoylglutathione (Sigma L7140) were monitored in a 96-well plate

17 for an increase in absorbance at $412 \mathrm{~nm}$. Reactions were pre-incubated at $37^{\circ} \mathrm{C}$ and initiated

18 with the addition of GloB. The conversion of DTNB to the yellow colored substrate, TNB, by

19 glutathione produced by GloB, was measured through time at $37^{\circ} \mathrm{C}$ and $412 \mathrm{~nm}$. Assays were

20 carried out over a range of GloB concentrations to ensure that the reaction rates are linear over

21 the period of the assay. Assays were performed using $\mathrm{Zn}^{+2}, \mathrm{Mn}^{+2}, \mathrm{Mg}^{+2}, \mathrm{Co}^{+2}$, and $\mathrm{Ca}^{+2}$.

23 Sample preparation for GloB vs. POM-ERJ mass spectrometry analysis. Reactions

24 containing $25 \mathrm{mM}$ Tris $\mathrm{HCl}(\mathrm{pH}$ 7.5), $250 \mathrm{mM} \mathrm{NaCl}, 10 \%$ glycerol, $1 \mathrm{mM} \mathrm{MnCl}$, and $1 \mathrm{mM}$ POM-

25 ERJ were pre-warmed to $37^{\circ} \mathrm{C}$ before addition of WT GloB, catalytically inactive GloB (H54N),

26 boiled GloB, or an equal amount of protein storage buffer to a final concentration of $1 \mu \mathrm{M}$. 
1 Reactions were placed at $37^{\circ} \mathrm{C}$ and sampled at $0,15,30,60,90$, and $120 \mathrm{~min}$. A $50 \mu \mathrm{L}$ sample

2 was withdrawn from each reaction at the times indicated, and the sample reaction was

3 quenched by the addition of $200 \mu \mathrm{L}$ acetonitrile containing $100 \mathrm{ng} / \mu \mathrm{L}$ enalapril as an internal

4 standard. The samples were immediately frozen on dry ice and stored at $-80^{\circ} \mathrm{C}$ until analysis.

6 The quenched reaction mixtures were centrifuged at $3200 \mathrm{rpm}$ for $5 \mathrm{~min}$, and $2 \mu \mathrm{L}$ of the

7 supernatant was diluted to $500 \mu \mathrm{L}$ with water containing $100 \mathrm{ng} / \mathrm{mL}$ enalapril as an internal

8 standard. Samples were analyzed by LC-MS/MS using an Applied Biosystems-Sciex API 4000.

9 Analyte/internal standard peak area ratios were used to determine concentration and evaluate

10 stability. Standards were evaluated over the range of $1 \mathrm{ng} / \mathrm{mL}$ to $1000 \mathrm{ng} / \mathrm{mL}$. The MRM

11 transitions for enalapril and POM-ERJ were m/z: $376.9>91.2$ and 424.0 > 364.0, respectively.

12 A Phenomenex Luna Omega polar C18 column $(2.1 \times 50 \mathrm{~mm}, 5 \mu \mathrm{m})$ was used for

13 chromatographic separation. Mobile phases were $0.1 \%$ formic acid in water and acetonitrile with

14 a flow rate of $0.5 \mathrm{~mL} / \mathrm{min}$. The starting phase was $1 \%$ acetonitrile increased to $100 \%$ acetonitrile

15 over 0.9 min. Peak areas were integrated using Analyst Software (AB Sciex, Foster City, CA).

17 In vivo cleavage of POM-ERJ. S. schleiferi cultures of WT and POM-ERJR strains were grown

18 to an $\mathrm{OD}_{600}=0.5-0.8$ and then treated with $1 \mu \mathrm{M}$ of POM-ERJ. The cultures were grown

19 shaking at $37^{\circ} \mathrm{C}$ and $200 \mathrm{rpm}$ and $50 \mu \mathrm{L}$ were sampled at $0,1,2$, and $3 \mathrm{~h}$. The reactions were

20 quenched by pelleting the cells at $4274 \times \mathrm{g}$ at $4^{\circ} \mathrm{C}$ and resuspending in $200 \mu \mathrm{L}$ of acetonitrile

21 with $100 \mathrm{ng} / \mathrm{\mu L}$ enalapril as an internal standard. The reactions were repeated in triplicate for

22 each timepoint and strain. The LC-MS analysis was performed as described above.

\section{Acknowledgments}

25 The authors are grateful to Joe Jez for ongoing support and helpful discussions. Financial 26 support provided by NIH Al123433 to CSD and the GWU Department of Chemistry. A.O.J. is 
bioRxiv preprint doi: https://doi.org/10.1101/2020.07.23.214460; this version posted July 23, 2020. The copyright holder for this preprint (which was not certified by peer review) is the author/funder. All rights reserved. No reuse allowed without permission.

1 supported by NIH/NIAID R01-AI103280, R21-Al123808, and R21-Al130584, and AOJ is an

2 Investigator in the Pathogenesis of Infectious Diseases (PATH) of the Burroughs Wellcome

3 Fund. 


\section{References}

2 1. Hsu J (2020) How COVID-19 is accelerating the threat of antimicrobial resistance. BMJ 3 1983(May):m1983.

42 Antimicrobial resistance in the age of COVID-19 (2020) Nat Microbiol 5(6):779-779.

5 3. Jindal A, Shivpuri D, Sood S (2015) Staphylococcus schleiferi meningitis in a child. $6 \quad$ Pediatr Infect Dis J 34(3):329.

7 4. Somayaji R, Rubin JE, Priyantha MA, Church D (2016) Exploring Staphylococcus

8 pseudintermedius: an emerging zoonotic pathogen? Future Microbiol 11(11):1371-1374.

9 5. Börjesson S, Gómez-Sanz E, Ekström K, Torres C, Grönlund U (2015) Staphylococcus

10 pseudintermedius can be misdiagnosed as Staphylococcus aureus in humans with dog

11 bite wounds. Eur J Clin Microbiol Infect Dis 34(4):839-44.

12 6. Lainhart W, Yarbrough ML, Burnham CA (2018) The brief case: Staphylococcus intermedius group-look what the dog dragged In. J Clin Microbiol 56(2). doi:10.1128/JCM.00839-17.

15 7. CDC (2019) Antibiotic resistance threats in the United States 2019. Available at: https://www.cdc.gov/drugresistance/pdf/threats-report/2019-ar-threats-report-508.pdf.

17 8. Fitzgerald JR (2009) The Staphylococcus intermedius group of bacterial pathogens: species re-classification, pathogenesis and the emergence of methicillin resistance. Vet Dermatol 20(5-6):490-5.

9. Humphries RM, et al. (2016) In vitro antimicrobial susceptibility of Staphylococcus pseudintermedius isolates of human and animal origin. J Clini 54(5):1391-1394.

10. Beever L, et al. (2015) Increasing antimicrobial resistance in clinical isolates of Staphylococcus intermedius group bacteria and emergence of MRSP in the UK. Vet Rec $176(7): 172$.

25 11. Haag NL, Velk KK, Wu C (2012) Potential antibacterial targets in bacterial central metabolism. Int J Adv life Sci 4(1-2):21-32. 
1 12. Murima P, McKinney JD, Pethe K (2014) Targeting bacterial central metabolism for drug 2 development. Chem Biol 21(11):1423-32.

3 13. Azema L, Baron R, Ladame S (2006) Targeting enzymes with phosphonate-based inhibitors: mimics of tetrahedral transition states and stable isosteric analogues of phosphates. Curr Enzym Inhib 2(1):61-72.

14. Kornberg RD, McNamee MG, McConnell HM (1972) Measurement of transmembrane potentials in phospholipid vesicles. Proc Natl Acad Sci U S A 69(6):1508-13.

8 15. Ju L, Cheng Z, Fast W, Bonomo RA, Crowder MW (2018) The continuing challenge of metallo- $\beta$-lactamase inhibition: mechanism matters. Trends Pharmacol Sci:1-13.

16. McKenney ES, et al. (2012) Lipophilic prodrugs of FR900098 are antimicrobial against Francisella novicida in vivo and in vitro and show GIpT independent efficacy. PLoS One 7(10):e38167.

17. Zhang $\mathrm{Y}$, et al. (2006) Activity of nitrogen-containing and non-nitrogen-containing bisphosphonates on tumor cell lines. J Med Chem 49(19):5804-14.

18. Hsiao C-HC, et al. (2014) Synthesis of a phosphoantigen prodrug that potently activates Vy9Vס2 T-lymphocytes. Chem Biol 21(8):945-954.

19. Wiemer AJ, Wiemer DF (2015) Prodrugs of phosphonates and phosphates: crossing the membrane barrier. 200(1):115-160.

20. Hecker SJ, Erion MD (2008) Prodrugs of phosphates and phosphonates. J Med Chem 51(8):2328-2345.

2121 Heidel KM, Dowd CS (2019) Phosphonate prodrugs: an overview and recent advances. Future Med Chem 11(13):1625-1643.

23 22. Sakagami K, et al. (1990) Synthesis and oral activity of ME 1207, a new orally active cephalosporin. J Antibiot (Tokyo) 43(8):1047-1050.

25 23. Siegel D, et al. (2017) Discovery and synthesis of a phosphoramidate prodrug of a pyrrolo[2,1- f][triazin-4-amino] adenine $C$-nucleoside (GS-5734) for the treatment of Ebola 
and emerging viruses. J Med Chem 60(5):1648-1661.

24. Shaw JP, et al. (1997) Metabolism and pharmacokinetics of novel oral prodrugs of 9-[(R)2-(phosphonomethoxy)propyl]adenine (PMPA) in dogs. Pharm Res 14(12):1824-9.

26. Sofia MJ, et al. (2010) Discovery of a $\beta$ - $d$-2'-deoxy-2'- $\alpha$-fluoro-2'- $\beta$ - C-methyluridine nucleotide prodrug (PSI-7977) for the treatment of Hepatitis C virus. J Med Chem

27. Edwards RL, et al. (2020) Potent, specific MEPicides for treatment of zoonotic staphylococci. PLoS Pathog 16(6):e1007806.

28. Uh E, et al. (2011) Antibacterial and antitubercular activity of fosmidomycin, FR900098, and their lipophilic analogs. Bioorg Med Chem Lett 21(23):6973-6976.

29. Edwards RL, et al. (2017) MEPicides: potent antimalarial prodrugs targeting isoprenoid biosynthesis. Sci Rep (June):1-11.

30. San Jose G, et al. (2013) Design of potential bisubstrate inhibitors against Mycobacterium tuberculosis (Mtb) 1-deoxy-D-xylulose 5-phosphate reductoisomerase (Dxr)—evidence of a novel binding mode. Medchemcomm:1099-1104.

31. San Jose G, et al. (2016) Structure-activity relationships of the MEPicides: N-acyl and Olinked analogs of FR900098 as inhibitors of DXR from Mycobacterium tuberculosis and Yersinia pestis. ACS Infect Dis 2(12):923-935.

23 32. Lin Y, et al. (2018) Eradication of ENO1-deleted glioblastoma through collateral lethality. bioRxiv. doi:10.1101/331538.

25 33. Erion MD, et al. (2005) Liver-targeted drug delivery using HepDirect prodrugs. $J$ Pharmacol Exp Ther 312(2):554-60. 
1 34. Sakamoto Y, Furukawa S, Ogihara H, Yamasaki M (2003) Fosmidomycin resistance in adenylate cyclase deficient (cya) mutants of Escherichia coli. Biosci Biotechnol Biochem 67(9):2030-2033.

4 35. Mackie RS, McKenney ES, van Hoek ML (2012) Resistance of Francisella Novicida to fosmidomycin associated with mutations in the glycerol-3-phosphate transporter. Front

36. Cui L, et al. (2003) Cell wall thickening is a common feature of vancomycin resistance in Staphylococcus aureus. J Clin Microbiol 41(1):5-14.

9 37. Jones T, et al. (2016) Failures in clinical treatment of Staphylococcus aureus infection with daptomycin are associated with alterations in surface charge, membrane

38. Choi Y, Sims GE, Murphy S, Miller JR, Chan AP (2012) Predicting the functional effect of amino acid substitutions and indels. PLoS One 7(10):e46688.

39. Misic AM, Cain CL, Morris DO, Rankin SC, Beiting DP (2014) Divergent isoprenoid biosynthesis pathways in Staphylococcus species constitute a drug target for treating infections in companion animals. mSphere 1(5):1-11.

40. Grosser MR, Richardson AR (2016) Method for preparation and electroporation of $S$. aureus and S. epidermidis. Methods Mol Biol 1373:51-7.

41. Nair D, et al. (2011) Whole-genome sequencing of Staphylococcus aureus strain RN4220, a key laboratory strain used in virulence research, identifies mutations that affect not only virulence factors but also the fitness of the strain. J Bacteriol 193(9):2332-

43. Doron S, et al. (2018) Systematic discovery of antiphage defense systems in the 5.

42. Schneewind O, Model P, Fischetti VA (1992) Sorting of protein A to the staphylococcal cell wall. Cell 70:267-281. microbial pangenome. Science 359(6379):eaar4120. 
$144 . \quad$ Puyet A, et al. (1987) A simple medium for rapid regeneration of Bacillus subtilis protoplasts transformed with plasmid DNA. FEMS Microbiol Lett 40(1):1-5.

3 45. Costa SK, Donegan NP, Corvaglia A-R, François P, Cheung AL (2017) Bypassing the restriction system to improve transformation of Staphylococcus epidermidis. J Bacteriol 199(16). doi:10.1128/JB.00271-17.

46. Monk IR, Tree JJ, Howden BP, Stinear TP, Foster TJ (2015) Complete bypass of restriction systems for major Staphylococcus aureus lineages. mBio 6(3).

47. Waterhouse A, et al. (2018) SWISS-MODEL: homology modelling of protein structures and complexes. Nucleic Acids Res 46(W1):W296-W303.

48. Yamamura A, et al. (2009) Structure of TTHA1623, a novel metallo- $\beta$-lactamase superfamily protein from Thermus thermophilus HB8. Acta Crystallogr Sect F Struct Biol Cryst Commun 65(Pt 5):455-9.

49. Melino S, Capo C, Dragani B, Aceto A, Petruzzelli R (1998) A zinc-binding motif conserved in glyoxalase II, $\beta$-lactamase and arylsulfatases. Trends Biochem Sci

50. Stamp AL, et al. (2010) Structural and functional characterization of Salmonella enterica serovar typhimurium YcbL: An unusual type II glyoxalase. Protein Sci 19(10):1897-1905.

51. Zang TM, Hollman DA, Crawford PA, Crowder MW, Makaroff CA (2001) Arabidopsis glyoxalase II contains a zinc/iron binuclear metal center that is essential for substrate binding and catalysis. J Biol Chem 276(7):4788-4795.

52. Park H-S, et al. (2006) Design and evolution of new catalytic activity with an existing protein scaffold. Science 311(5760):535-8.

24 53. Murata K, et al. (1986) Metabolism of alpha-ketoaldehydes in yeasts: Purification and characterization of glyoxalase II from Saccharomyces cerevisiae. Agric Biol Chem 50(1):135-142. 
1 54. Irsch T, Krauth-Siegel RL (2004) Glyoxalase II of African trypanosomes is trypanothionedependent. J Biol Chem 279(21):22209-22217.

3 55. Norton SJ, Talesa V, Yuan WJ, Principato GB (1990) Glyoxalase I and glyoxalase II from Aloe vera: purification, characterization and comparison with animal glyoxalases. Biochem Int 22(3):411-418.

56. Norton SJ, Principato GBB, Talesa V, Lupattelli M, Rosi G (1989) Glyoxalase II from Zea mays: properties and inhibition study of the enzyme purified by use of a new affinity

57. Talesa V, et al. (1990) Presence of glyoxalase II in mitochondria from spinach leaves: comparison with the enzyme from cytosol. Biochem Int 22(6):1115-20.

58. Talesa V, et al. (1990) Isolation of glyoxalase II from bovine liver mitochondria. Biochem Int 20(1):53-8.

59. vander Jagt D. (1989) The glyoxalase system. Chem Biochem Med Asp:597-641.

60. Cho MY, Bae CD, Park JB, Lee TH (1998) Purification and cloning of glyoxalase II from rat liver. Exp Mol Med 30(1):53-7.

61. Uotila L (1973) Purification and characterization of S-2-hydroxyacylglutathione hydrolase (glyoxalase II) from human liver. Biochemistry 12:3944-3951.

62. Schrödinger The PyMOL molecular graphics system. Available at: https://pymol.org/2/.

63. Cameron AD, Ridderström M, Olin B, Mannervik B (1999) Crystal structure of human glyoxalase II and its complex with a glutathione thiolester substrate analogue. Structure 7(9):1067-1078.

64. Brown SA, Palmer KL, Whiteley M (2008) Revisiting the host as a growth medium. Nat Rev Microbiol 6(9):657-666.

24 65. Fey PD, et al. (2013) A genetic resource for rapid and comprehensive phenotype screening of nonessential Staphylococcus aureus genes. mBio 4(1). doi:10.1128/mBio.00537-12. 
66. Baba T, et al. (2006) Construction of Escherichia coli K-12 in-frame, single-gene knockout mutants: the Keio collection. Mol Syst Biol 2(1). doi:10.1038/msb4100050.

67. Joyce AR, et al. (2006) Experimental and computational assessment of conditionally essential genes in Escherichia coli. J Bacteriol 188(23):8259-8271.

68. Armstrong CM, Meyers DJ, Imlay LS, Freel Meyers C, Odom AR (2015) Resistance to the antimicrobial agent fosmidomycin and an FR900098 prodrug through mutations in the deoxyxylulose phosphate reductoisomerase gene (dxr). Antimicrob Agents Chemother 59(9):5511-5519.

69. Pines G, et al. (2018) Genomic deoxyxylulose phosphate reductoisomerase (DXR)

12 70. Koul A, et al. (2007) Diarylquinolines target subunit c of mycobacterial ATP synthase. Nat Chem Biol 3(6):323-324.

14 71. Haagsma AC, et al. (2009) Selectivity of TMC207 towards Mycobacterial ATP synthase compared with that towards the Eukaryotic homologue. Antimicrob Agents Chemother

72. Andries K (2005) A diarylquinoline drug active on the ATP synthase of Mycobacterium tuberculosis. Science 307(5707):223-227.

73. Gomes RA, et al. (2006) Yeast protein glycation in vivo by methylglyoxal. Molecular modification of glycolytic enzymes and heat shock proteins. FEBS J 273(23):5273-87.

21 74. Rahman A, Shahabuddin, Hadi SM (1990) Formation of strand breaks and interstrand cross-links in DNA by methylglyoxal. J Biochem Toxicol 5(3):161-166.

75. Migliore L, et al. (1990) Genotoxicity of methylglyoxal: cytogenetic damage in human lymphocytes in vitro and in intesting cells of mice. Carcinogenesis 11(9):1503-1507.

25 76. Hayes G, et al. (2018) Manuka honey and methylglyoxal increase the sensitivity of 
1 77. Müller P, et al. (2013) Synergism between Medihoney and rifampicin against methicillinresistant Staphylococcus aureus (MRSA). PLoS One 8(2):e57679.

3 78. Bulman SEL, Tronci G, Goswami P, Carr C, Russell SJ (2017) Antibacterial properties of nonwoven wound dressings coated with manuka honey or methylglyoxal. Mater (Basel, Switzerland) 10(8). doi:10.3390/ma10080954.

6 79. Paramasivan S, et al. (2014) Methylglyoxal-augmented manuka honey as a topical antiStaphylococcus aureus biofilm agent: safety and efficacy in an in vivo model. Int Forum

80. Determination of minimum inhibitory concentrations (MICs) of antibacterial agents by broth dilution (2003) Eur Soc Clin Microbiol Infect Dis (March):1-7.

11 81. Li H, et al. (2009) The sequence alignment/map format and SAMtools. Bioinformatics 25(16):2078-9.

82. Danecek P, et al. (2011) The variant call format and VCFtools. Bioinformatics 27(15):2156-8.

83. Cingolani $\mathrm{P}$, et al. (2012) A program for annotating and predicting the effects of single nucleotide polymorphisms, SnpEff: SNPs in the genome of Drosophila melanogaster

84. Alexandrov A, et al. (2004) A facile method for high-throughput co-expression of protein pairs. Mol Cell Proteomics 3(9):934-8.

85. O'Callaghan $\mathrm{CH}$, Morris A, Kirby SM, Shingler AH (1972) Novel method for detection of $\beta$-lactamases by using a chromogenic cephalosporin substrate. Antimicrob Agents Chemother 1(4):283-8.

86. Yatsunenko T, et al. (2012) Human gut microbiome viewed across age and geography. Nature 486(7402):222-227.

87. Madeira F, et al. (2019) The EMBL-EBI search and sequence analysis tools APIs in 
bioRxiv preprint doi: https://doi.org/10.1101/2020.07.23.214460; this version posted July 23, 2020. The copyright holder for this preprint (which was not certified by peer review) is the author/funder. All rights reserved. No reuse allowed without permission.

1 88. Letunic I, Bork P (2019) Interactive Tree Of Life (iTOL) v4: recent updates and new 2 developments. Nucleic Acids Res 47(W1):W256-W259.

3 